\title{
Waves and Magnetism in the Solar Atmosphere (WAMIS)
}

\author{
L. $\operatorname{Strachan}^{1} \dagger$, Y.-K. Ko ${ }^{1}$, J. D. Moses ${ }^{1}$, J. M. Laming ${ }^{1}$, F. Auchere ${ }^{2}$, \\ R. Casini $^{3}$, S. Fineschi ${ }^{4}$, S. Gibson ${ }^{3}$, M. Knoelker ${ }^{3}$, C. Korendyke ${ }^{1}$, \\ S. Mcintosh ${ }^{3}$, M. Romoli ${ }^{5}$, J. Rybak ${ }^{6}$, D. Socker ${ }^{1}$, S. Tomczyk ${ }^{3}$, \\ A. Vourlidas ${ }^{1} \ddagger$ and $\mathrm{Q}$. $\mathbf{W u}^{3}$ \\ ${ }^{1}$ Naval Research Laboratory, Washington, DC 20375, USA \\ ${ }^{2}$ Institut d'Astrophysique Spatiale, 91405 Orsay, France \\ ${ }^{3}$ High Altitude Observatory/NCAR, Boulder, CO 80301, USA \\ ${ }^{4}$ INAF-Astrophysical Observatory of Turin, 10025 Pino Torinese TO, Italy \\ ${ }^{5}$ University of Florence, 50121, Florence, Italy \\ ${ }^{6}$ Astronomical Institute of the Slovak Academy of Sciences, 05960 Tatranská Lomnica, The \\ Slovak Republic \\ email: leonard.strachan@nrl.navy.mil
}

\begin{abstract}
Magnetic fields in the solar atmosphere provide the energy for most varieties of solar activity, including high-energy electromagnetic radiation, solar energetic particles, flares, and coronal mass ejections, as well as powering the solar wind. Despite the fundamental role of magnetic fields in solar and heliospheric physics, there exist only very limited measurements of the field above the base of the corona. What is needed are direct measurements of not only the strength and orientation of the magnetic field but also the signatures of wave motions in order to better understand coronal structure, solar activity, and the role of MHD waves in heating and accelerating the solar wind. Fortunately, the remote sensing instrumentation used to make magnetic field measurements is also well suited to measure the Doppler signature of waves in the solar structures. We present here a mission concept for the Waves And Magnetism In the Solar Atmosphere (WAMIS) experiment which is proposed for a NASA long-duration balloon flight.
\end{abstract}

Keywords. Sun: magnetic fields, Sun: corona, balloons, instrumentation: polarimeters

\section{Introduction}

WAMIS is the Waves And Magnetism In the Solar Atmosphere mission proposed for the NASA Low Cost Access to Space program. It is designed to make direct solar coronal measurements of not only the strength and orientation of the magnetic field, but also the signatures of wave motions in order to better understand coronal structure, solar activity, and the role of MHD waves in heating and accelerating the solar wind. WAMIS will take advantage of greatly improved infrared detectors, forward models, advanced diagnostic tools, and inversion codes to obtain a breakthrough in the measurement of coronal magnetic fields and in the understanding of the interaction of these fields with space plasmas. This will be achieved with a high altitude balloon borne payload consisting of a coronagraph with an IR spectro-polarimeter focal plane assembly. Operating from an altitude of $\sim 35 \mathrm{~km}$, the balloon platform provides minimum atmospheric absorption and scattering at the IR wavelengths in which these observations are made.

$\dagger$ also a Smithsonian Research Associate

$\ddagger$ now at Johns Hopkins University/Applied Physics Laboratory 

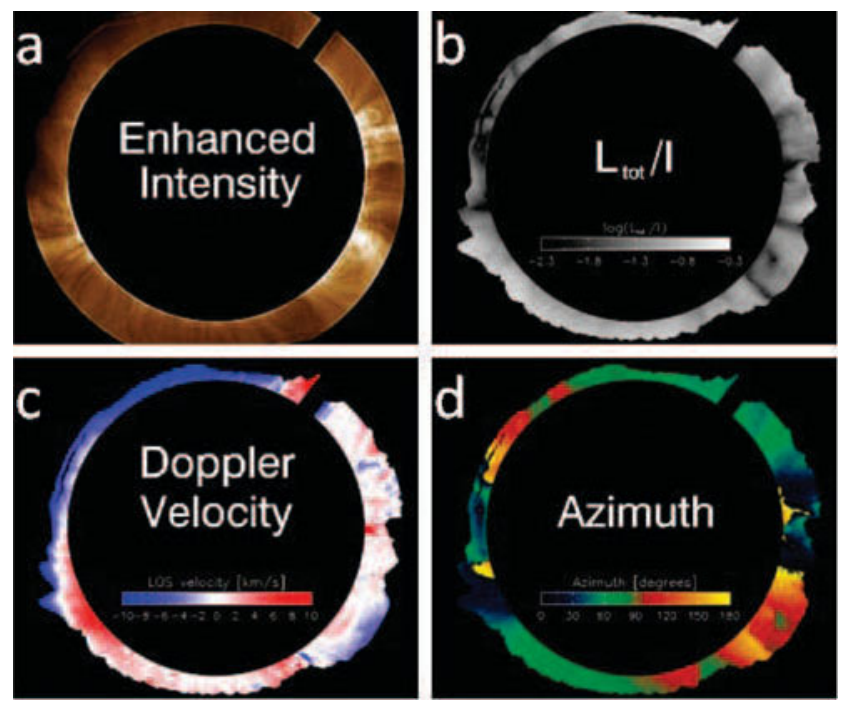

Figure 1. Sample CoMP Fe XIII $1074.7 \mathrm{~nm}$ images showing a magnetic loop at the SW limb on 28 September 2012. Images are shown for the following: a) total intensity I, b) linear polarization $\left(L / I=\sqrt{Q^{2}+U^{2}} / I\right)$, c) Doppler velocity from line shifts, and d) field azimuth angle $(\psi=1 / 2 \arctan (U / Q))$. WAMIS will produce similar data over a wider field of view, but with higher spatial resolution and sensitivity to the magnetic field.

In the following sections, we present the science objectives, measurement strategies, and instrument properties for the proposed experiment. Here, we emphasize the polarimetric capabilities for determining the magnetic field strength and its direction in the corona, although it should be understood that WAMIS will be capable of measuring the properties of propagating waves as well.

\section{Science Objectives}

The four primary objectives of WAMIS are aimed to improve our understanding of the role of magnetic fields and waves in determining the properties of the solar wind, coronal mass ejections (CMEs) and flares, magnetic current sheets, and shock fronts which are often associated with the fastest CMEs.

Objective 1: What determines the magnetic structure of the corona? The magnetic field not only shapes the corona on all size scales from bright points to active regions and streamers, it also modulates the structure of the solar wind streams produced by the Sun. The turbulence and waves in these loops may also be responsible for the first ionization potential (or FIP) fractionation of the slow solar wind (see the review by Laming 2014). WAMIS will be able to determine the magnetic field geometry in the corona and directly detect the existence of waves by building on the work of Tomczyk et al. (2007). Their results were based on data from the Coronal Multichannel Polarimeter (CoMP) instrument, whose design is incorporated into WAMIS. Representative CoMP images showing coronal structures off the solar limb are shown in Figure 1.

Objective 2: How do flux ropes form and evolve, and how are they related to CMEs? Whether CMEs are formed by twisted magnetic fields called flux ropes is still a contentious topic in solar physics. However observations of quiescent prominences in emission lines seem to show twisted strands that appear to follow the magnetic field. It is not entirely clear how the initial magnetic structure of the prominence evolves into 
a flux rope prior to a CME. WAMIS can provide the needed information by mapping the magnetic field in coronal loops and prominences (e.g. see Rachmeler et al. 2013 and Bak-Steślika et al. 2013). The measurements from WAMIS will also put constraints on the plasma density, stored magnetic energy, and magnetic helicity.

Objective 3: Where do CME-associated shocks form? Vourlidas et al. (2003) used SOHO/LASCO white light observations to reveal what appears to be a density enhancement at a CME shock below $2.5 \mathrm{R}_{\odot}$ in the corona. With measurements of the magnetic field strength and direction from WAMIS, firm constraints can be put on the shock formation height, its Alfvén Mach number, and its obliquity. Whether a shock is quasi-parallel or quasi-perpendicular has consequences for particle acceleration mechanisms.

Objective 4: How is energy stored and released by reconnection in flares and CMEs? Magnetic reconnection is thought to be involved in the impulsive release of energy by flares. Large CME eruptions often show a high temperature region which appears to connect the CME to the flaring region at the base of the corona. These regions have been observed with SOHO/UVCS using the Fe XVIII $97.5 \mathrm{~nm}$ line by Ko et al. (2003) and Ciaravella \& Raymond (2008). WAMIS can be used to discriminate between various models by determining the magnetic topology before and after current sheet formation. Also, WAMIS should be able to estimate the amount of energy in the current sheet from a determination of the magnetic field strength.

\section{Measurement Strategy}

A NASA long duration balloon flight mission from Antarctica can achieve continuous observations over most of a solar rotation, covering all of the key time scales for the evolution of coronal magnetic fields. In particular, a balloon flight will make it possible to do the following: i) remove the variability in the sky polarization background; ii) improve the overall stray light level by reducing the sky brightness background; and iii) observe without interruptions from day-night cycles or weather. The last point will provide better temporal continuity with uninterrupted observations for at least two weeks. This will increase the probability for observing CMEs without interruptions. Finally, the observations also allow for tomographic reconstructions, which will help in separating the 3D structure of the corona from its short-term temporal evolution.

Table 1 shows the expected values for the coronal field of view (measured from Suncenter), spatial resolution, and the physical observables that will be used to address the above science questions. The physical observables to be obtained by WAMIS are listed in the last column of Table 1 and they are explained in Table 2.

WAMIS will measure the polarization of three infrared lines, off the limb, in order to constrain values for the strength and direction of the coronal magnetic field. The primary emission lines that were selected are the Fe XIII lines at 1074.7 and $1079.8 \mathrm{~nm}$ and the He I line at $1083.0 \mathrm{~nm}$. These lines were chosen for their usefulness in Hanle effect measurements (e.g., see Trujillo Bueno 2014) and as a density diagnostic (using the Fe XIII line ratio). The Hanle effect is a modification of the polarization for a resonance emission line due to the presence of a magnetic field. In general, both the strength and direction of the magnetic field can be determined. However, for the Fe XIII forbidden lines and for the He I line (with $|\mathbf{B}|>10 \mathrm{G}$ ), the effect is saturated and only the field direction can be determined. The field direction can be inferred from linear polarization measurements to determine the I, Q, and U Stokes parameters. Circular polarization measurements (longitudinal Zeeman effect) are required to determine the magnitude of 
Table 1. Observational Requirements

\begin{tabular}{|l|c|c|}
\hline Science Objective & FoV /Spatial Resolution & Physical Observable \\
\hline \begin{tabular}{l|c|c|} 
1. Fast/Slow Wind, \\
Coronal B structure
\end{tabular} & $\begin{array}{c}1.02-1.8 R_{\odot} / 1.5 " \text { pix. } \\
1.02-2.8 R_{\odot} / 4.5 " \text { pix. }\end{array}$ & $\begin{array}{c}\text { Waves: Doppler velocity, B-field } \\
\text { direction, plasma density }\end{array}$ \\
\hline \begin{tabular}{l|c|c|} 
2. Prominences, \\
flux ropes
\end{tabular} & $1.02-1.8 R_{\odot} / 1.5 "$ pix & $\begin{array}{c}\text { B-field magnitude \& direction from He I } \\
\text { and Fe XIII }\end{array}$ \\
\hline 3. CME Shocks & $1.02-2.8 R_{\odot} / 4.5 "$ pix. & $\begin{array}{c}\text { B-field magnitude \& direction, plasma } \\
\text { density; Waves: Doppler velocity }\end{array}$ \\
\hline 4. Reconnection & $1.02-1.8 R_{\odot} / 1.5 "$ pix. & $\begin{array}{c}\text { B-field magnitude \& direction, } \\
\text { Waves: Doppler velocity, plasma density }\end{array}$ \\
\hline
\end{tabular}

Table 2. Key WAMIS Observables

\begin{tabular}{|l|c|c|}
\hline Observable & Analysis Method & Physical Process \\
\hline Line-of-Sight B-field Strength & Circular Polarization & Long. Zeeman Effect \\
\hline Plane-of-Sky B-field Direction & Linear Polarization & $\begin{array}{c}\text { Resonance Scattering \& } \\
\text { (Saturated) Hanle Effect }\end{array}$ \\
\hline Line-of-Sight Velocity & Intensity vs. Wavelength & Doppler Effect \\
\hline Plasma Density & $\begin{array}{c}\text { Fe XIII 1074 nm/1079.8 nm } \\
\text { Int. Ratio; K-corona from continum }\end{array}$ & $\begin{array}{c}\text { Atomic Physics, } \\
\text { Radiation Transfer }\end{array}$ \\
\hline
\end{tabular}

the field projected along the line of sight. WAMIS will measure the circular polarization of the Fe XIII lines. It will be capable of determining LOS field strengths down to $2 \mathrm{G}$ in 5 min observations (see Figure 2), assuming Stokes $\mathrm{V}$ amplitudes are on the order of $\sim 10^{-4}$.

\section{Instrument Description}

WAMIS has a classical Lyot coronagraph design, consisting of a $20 \mathrm{~cm}$ objective lens, a field lens with an internal occulter, and a Lyot stop to block the diffraction from the edges of the objective lens. A collimating lens is used to collimate the light before it passes through a polarimeter/filter. Light from the polarizer is then reimaged onto a $1280 \times 1024$ format InGaAs detector. Table 3 presents the relevant characteristics of the WAMIS instrument.

The stray light properties of the instrument will be achieved with low-scatter optical materials and following the same cleanliness practices performed with previous NRL coronagraphs, e.g. Howard et al. (2008).

The WAMIS filter/polarimeter has direct heritage from the existing Coronal Multichannel Polarimeter (CoMP) instrumentation, described by Tomczyk et al. (2008). CoMP has been making coronal polarization measurements from the Mauna Loa Solar Observatory (3,398 m altitude) since 2011 (see Fig. 1 for sample images). However, WAMIS will have several design improvements over CoMP, making it a more capable instrument for measuring weak coronal magnetic fields.

The CoMP polarimeter has a 4-stage combination of Lyot filters, polarizers, and halfwaveplate retarders to optimize the polarization signals for the Fe XIII lines. Instead of the liquid crystal variable retarders (LCVRs) used by CoMP, WAMIS will use Ferroelectric Liquid Crystal (FLC) retarders. The FLC optics have a much faster response time and will provide a broader spectral range for WAMIS. In addition to the three primary lines in Table 3, WAMIS will be capable of observing Fe lines in corona (530.3 Fe XIV, 
Table 3. WAMIS Instrument Characteristics

\begin{tabular}{|c|c|}
\hline Telescope Type & Internally Occulted Lyot Coronagraph \\
\hline Objective Lens (type, aperture size, focal length) & f/10 singlet, $20 \mathrm{~cm}, 203.3 \mathrm{~cm}$ \\
\hline Objective Stray light & $<0.2 \mu B_{\odot}\left(\right.$ goal for $\left.1.2-2.8 \mathrm{R}_{\odot}\right)$ \\
\hline Inner Field of View Limit & $1.02 \mathrm{R}_{\odot}$ \\
\hline Outer Field of View Limit & $\pm 2.8 \mathrm{R}_{\odot}$ at $4.5 " /$ pixel (Sun Centered) \\
\hline Filter (type, aperture size \& wavelength range) & Tunable Lyot filter, $3.8 \mathrm{~cm}, 530-10830 \mathrm{~nm}$ \\
\hline Primary Spectral Lines of Interest ${ }^{1}$ & $\begin{array}{c}\text { Fe XIII }(1074.7,1079.8 \mathrm{~nm}) \\
\text { Fe X }(637.5 \mathrm{~nm}) ; \text { He I }(1083.0 \mathrm{~nm})\end{array}$ \\
\hline Detector (mfr., pitch, format) & Goodrich camera, $15 \mu \mathrm{m}$ pixels, $1280 \times 1024$ \\
\hline Plate Scale (at low \& high magnification) & $4.5 " /$ pixel \& $1.5 " /$ pixel \\
\hline Overall Throughput & $\approx 5 \%$ \\
\hline Count Rate for Fe XIII $(1074.7 \mathrm{~nm})$ at $1.1 \mathrm{R}_{\odot}$ & $1 \times 10^{5}$ photons $/$ pixel/s at high magnification \\
\hline Duration of Continuous Observing Sequences & 2 weeks minimum ( $\geqslant 4$ weeks optimum) \\
\hline
\end{tabular}

${ }^{1}$ Note: Fe XIV 530.3, Fe X 637.5, Fe IX 789.2, He I $587.6 \& \mathrm{H} \alpha 656.3(\mathrm{~nm})$ are also observable

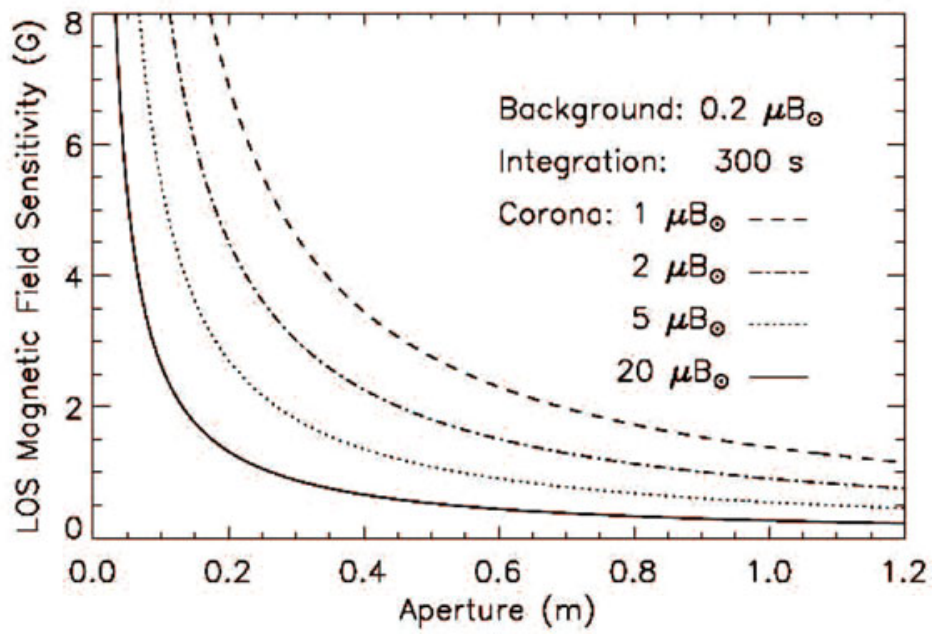

Figure 2. The magnetic field sensitivity (in G) vs Aperture (in m) for different values of brightness for the observed coronal feature. Brightness values are in units of the mean solar flux from the disk. WAMIS will have a $20 \mathrm{~cm}$ aperture and will be capable of making magnetic field measurements as low as $2 \mathrm{G}$ with 5 minute observations.

637.5 Fe X and 789.2 Fe XI); and also 587.6 He I and 656.3 $\mathrm{H} \alpha$ in prominences. Since WAMIS will be above most of the atmosphere, there is no need to simultaneously observe the continuum for background corrections and thus the beamsplitter used by CoMP can be removed from the optical train.

The detector for WAMIS has been upgraded from the one used by CoMP. Instead of a liquid nitrogen cooled $\mathrm{HgCdTe}$ detector, WAMIS will use an InGaAs Visible+short wavelength IR detector (Goodrich GA $1280 \mathrm{~J}$ ), operating at $-20^{\circ} \mathrm{C}$. The detector's thermoelectric (TEC) cooler will be coupled to a low temperature radiator on the balloon gondola to ensure low noise operation (NEI of $3.1 \times 10^{3}$ photons $/$ pixel $/ \mathrm{s}$ ). The quantum efficiency with this detector is near $80 \%$ for the primary wavelengths of interest. 


\section{Present Status and Future Work}

WAMIS has been submitted to the Low Cost Access to Space (LCAS) program and is awaiting the selection by NASA but in the meantime, several subsystems of the instrument and balloon gondola have already been tested. The new FLC and fixed retarders have been used in the CoMP-S filter/polarimeter on the Lomnicky Peak Observatory (2,632 $\mathrm{m}$ altitude) in Slovakia. Polarimetric observation at Lomnicky Peak have verified the wavelength performance and free spectral range $(0.50 \mathrm{~nm}$ at $530 \mathrm{~nm}$ and $2.34 \mathrm{~nm}$ at $1083 \mathrm{~nm}$ ) of the WAMIS filter will meet the science requirements.

WAMIS will use a gondola and payload pointing system provided by the Balloon Program Office at NASA/Wallops. The Wallops Arc Second Pointer (WASP) system has achieved a pointing stability of $0.15 \operatorname{arcsec}(\mathrm{rms})$ pitch error and $0.22 \operatorname{arcsec}(\mathrm{rms})$ yaw error during test flights from Fort Sumner, New Mexico. These values compare favorably to the 1.5 arcsec pixel requirement for the most stringent WAMIS observations.

Finally, to prepare for analyzing the data from WAMIS, we will use the FORWARD emission line modeling code by Gibson et al. (in these proceedings and Forland et al. 2013) to model the expected polarization signatures from simulated magnetic models and expected WAMIS instrument characteristics. Similar studies were made for prominence cavities observed with CoMP and reported by Bak-Steślika et al. (2013). This work will increase the confidence of the magnetic field determinations derived from WAMIS observations.

\section{Acknowledgements}

This work is funded by the Naval Research Laboratory basic research program and NASA through NDPR NNG13WF95I. CoMP images are courtesy of the Mauna Loa Solar Observatory, operated by the High Altitude Observatory, as part of the National Center for Atmospheric Research (NCAR). NCAR is supported by the National Science Foundation.

\section{References}

Bak-Steślika, U., Gibson, S. E., Fan, Y., Bethge, C., Forland, B., \& Rachmeler, L. A. 2013, ApJ $770, \mathrm{~L} 28$

Ciaravella, A. \& Raymond, J. C. 2008, ApJ 686, 1372

Dove, J. B., Gibson, S. E., Rachmeler, L. A., Tomczyk, S., \& Judge, P. 2011, ApJ 731, 1

Forland, B., Gibson, S. E., Dove, J., \& Kucera, T. 2013, in: Nature of Prominences and their role in Space Weather, Proc. IAU Symp S300 (Cambridge U.P.), p. 414

Howard, R. A., Moses, J. D., Vourlidas, A., Newmark, J. S., Socker, D. G., Plunkett, S. P., Korendyke, C. M., et al. 2008, Space Sci. Rev. 136, 67

Ko, Y.-K., Raymond, J. C., Lin, J., Lawrence, G., Li, J., \& Fludra, A. 2003, ApJ 594, 1068

Laming, J. M. 2014, Living Reviews of Solar Physics, submitted

Rachmeler, L. A., Gibson, S. E., Dove, J. B., DeVore, C. R., \& Fan, Y. 2013, Sol. Phys. 288, 617

Tomczyk, S., McIntosh, S. W., Keil, S. L., Judge, P. G., Schad, T., Seeley, D. H., \& Edmonson, J. 2007, Science 319, 1192

Tomczyk, S., Card, G. L., Darnell, T., Elmore, D. F., Lull, R., Nelson, P. G., Streander, K. V., Burkepile, J., Casini, R., \& Judge, P. G. 2008, Sol. Phys. 247, 411

Trujillo Bueno, J. 2014, in: K. N. Nagendra, J. O. Stenflo Z. Q. Qu, \& M. Sampoorna (eds.), Solar Polarization 7, ASP Conf. Series 489 (San Francisco: ASP), p. 137

Vourlidas, A., Wu, S. T., Wang, A. H.,Subramanian, P., \& Howard, R. A. 2003, ApJ 598, 1392 